\title{
PURE ROTATIONAL RAMAN LIDAR FOR TEMPERATURE MEASUREMENTS FROM 5-40 KM OVER WUHAN, CHINA
}

\author{
Yajuan $\mathrm{Li}^{1,2}$, Shalei Song ${ }^{1}$, Yong Yang ${ }^{1,3 *}$, Faquan $\mathbf{L i}^{1}$,Xuewu Cheng ${ }^{1}$, \\ Zhenwei Chen ${ }^{1}$, Linmei Liu ${ }^{1}$, M. Patrick McCormick ${ }^{3}$, Shunsheng Gong ${ }^{1}$ \\ ${ }^{1}$ Wuhan Institute of Physics and Mathematics, The Chinese Academy of Sciences, State Key Laboratory \\ of Magnetic Resonance and Atomic and Molecular Physics, Wuhan 430071, China \\ *Email: yangyong2019@sina. com \\ ${ }^{2}$ University of Chinese Academy of Sciences, Beijing 100049, China \\ ${ }^{3}$ Department of Atmospheric and Planetary Sciences, Center for Atmospheric Sciences, Hampton \\ University, Hampton, Virginia 23668, USA.
}

\begin{abstract}
In this paper a pure rotational Raman lidar (PRR) was established for the atmospheric temperature measurements from $5 \mathrm{~km}$ to $40 \mathrm{~km}$ over Wuhan, China $\left(30.5^{\circ} \mathrm{N}, 114.5^{\circ} \mathrm{E}\right)$. To extract the expected PRR signals and simultaneously suppress the elastically backscattered light, a high-spectral resolution polychromator for light splitting and filtering was designed. Observational results revealed that the temperature difference measured by PRR lidar and the local radiosonde below 30 $\mathrm{km}$ was less than $3.0 \mathrm{~K}$. The good agreement validated the reliability of the PRR lidar. With the 1-h integration and 150-m spatial resolution, the statistical temperature error for PRR lidar increases from $0.4 \mathrm{~K}$ at $10 \mathrm{~km}$ up to $4 \mathrm{~K}$ at altitudes of about $30 \mathrm{~km}$. In addition, the whole night temperature profiles were obtained for study of the long-term observation of atmospheric fluctuations.
\end{abstract}

\section{INTRODUCTION}

The temperature structure of the atmosphere plays a key role in understanding the dynamical, radiative and coupling processes of different atmospheric layers ${ }^{[1]}$. To study atmospheric energy transport and the impact on the environment from anthropogenic behaviors, it is of great significance to understand the connective detection of the Earth's atmospheric temperature over a wide vertical range.

To date, versatile lidar techniques have been developed for routine atmospheric temperature measurements with high precision, and high temporal and spatial resolution. Based on different methodologies, temperature profiles can be retrieved from the ground to the mesosphere and lower thermosphere (MLT). Based on the Rayleigh integration technique, absolute temperature above $\sim 35 \mathrm{~km}$ can be accurately deduced from the relative air density, assuming that the pure atmosphere is in hydrostatic equilibrium and obeys the ideal gas law ${ }^{[2]}$. When Mie scattering from aerosols gradually contaminate Rayleigh signals, a vibrational Raman lidar that depends on a specific frequency shift can be used instead to extend the temperature detection to lower altitudes ${ }^{[3]}$. The weaker cross section of vibrational Raman and the interference of the aerosols, clouds and ozone concentration present considerable experimental difficulty for high altitude and high precision detection. With a relatively stronger cross section than vibrational Raman, rotational Raman (RR) lidar is a candidate for high precision temperature measurements from the ground to the upper stratosphere, even in the presence of aerosols and optically-thick cloud layers ${ }^{[4]}$. With progress in technologies of spectral extraction and weak signal detection, the temperature detection altitude for RR lidar can be extended upward to the stratosphere ${ }^{[5,6]}$. However, it is still a challenge for the RR technique to provide temperature measurements up to above $30 \mathrm{~km}$ with high precision. A large power-aperture product and sufficient suppression of the stronger elastic backscattering (about $6-8$ orders of magnitude) are important steps to obtain signal-to-noise ratios (SNR) and precision that are required.

In this paper, a PRR lidar system with a highspectral resolution polychromator was built for high precision temperature measurements from 5- 
$40 \mathrm{~km}$ over Wuhan. Temperature measurements up to $40 \mathrm{~km}$ by PRR lidar showed the great potential for the further combination with Rayleigh lidar that would provide for study of the middle to lower atmosphere.

\section{METHODOLOGY}

The principle for PRR temperature measurements is mainly based on the fact that the intensities of molecular rotational Raman lines exhibit different dependencies on temperature ${ }^{[7]}$ as a result of the Maxwell-Boltzmann distribution. To determine the temperature, two domains (RR1 \& RR2) with opposite temperature sensitivities are extracted from the anti-Stokes branch of the RR spectrum. To suppress the elastic backscatter returns, stateof-the art interference filters are selected to realize the signal separation with high spectral resolution.

In our simulation, the central wavelengths (CWL) and the full-width-at-half-maximum bandwidths (FWHM) of the filters are the primary parameters taken into account. Two opposite temperature dependence regions are distinguished at the wavelength of $\sim 530.5 \mathrm{~nm}$. In terms of the minimum statistical temperature error, the optimal CWLs for the two RR channels are selected at $531.4 \mathrm{~nm}$ and $528.7 \mathrm{~nm}$ separately for the FWHMs of $0.7 \mathrm{~nm}$ and $1.1 \mathrm{~nm}^{[8]}$. The ratio of the detected signal in RR1 and RR2 channels is sensitive to temperature as shown in equation (1).

$$
\begin{aligned}
& Q T)=\frac{S_{R 2}(T)}{S_{R 21}(T)}=\exp \left(\frac{a}{T^{2}}+\frac{b}{T}+c\right) \\
& T=\frac{-2 a}{b \pm \sqrt{b^{2}-4 a(c-\ln Q)}} \\
& \Delta T=\frac{\partial T}{\partial Q} Q \cdot \sqrt{\frac{1}{S_{R 21}+\frac{1}{S_{R 2}}}}
\end{aligned}
$$

In Equations (1-3), $\mathrm{T}$ is the temperature; $\mathrm{Q}$ is the signal ratio; $S_{R 1}$ and $S_{F 22}$ are the detected signals in RR1 and RR2 channels; $a, b, c$ are the calibration coefficients; $\Delta T$ is the statistical error of RR temperature measurements. With the calibration function in Eq. (1) and the local radiosonde temperature data, the calibration state will be determined for a stable-working lidar system.

Fig. 1 shows the schematic overview of the PRR lidar system whose main characteristics are presented in Table 1. A frequency-doubled and seeded-injection Nd:YAG laser is used as a transmitter to emit light at $532.1 \mathrm{~nm}$ with a repetition rate of $30 \mathrm{~Hz}$. After expansion the laser beam with a divergence of $\sim 170 \mu \mathrm{rad}$ is directed vertically into the atmosphere.

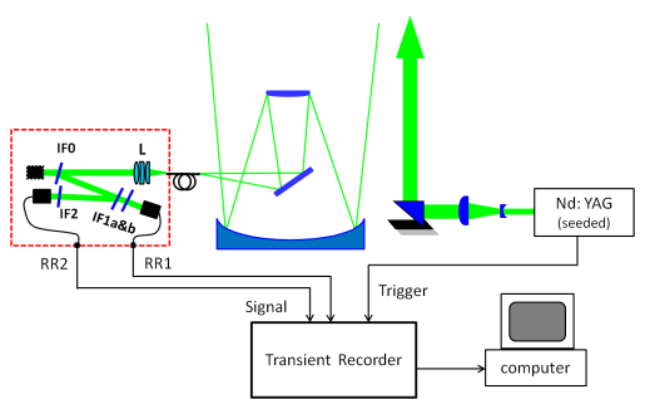

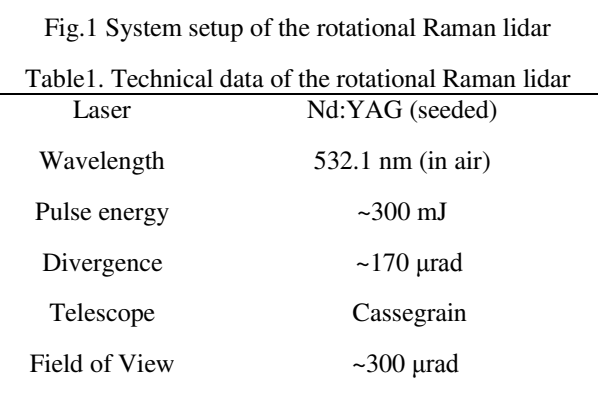

Signals backscattered from the atmosphere are collected by a Cassegrain telescope and transferred into the beam collimating and splitting system. Finally two RR signals are captured by a transient reorder (TR20-160, manufactured by Licel) and stored in a computer for the later data processing. Fig. 2 is a photo of the transmitter and receiver scene of the lidar system.

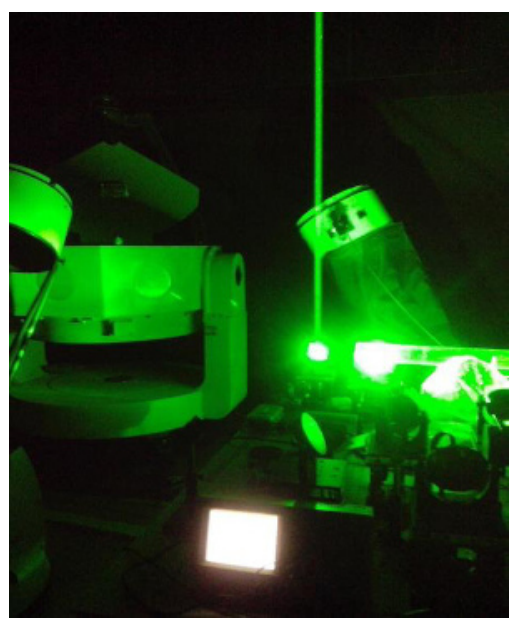

Fig.2 Transmitter and receiver of the rotational Raman lidar system 
Since filter performance changes with the angle of incidence, a higher level of collimation is demanded for the $1.5 \mathrm{~m} \mathrm{Z}$-shaped separating light path. Optical simulation shows that the collected backscattering can be coupled efficiently into the fiber. With a set of combined lenses for collimation, the beam spot size is confined within the area of the tilted interference filters. The transmission curves of the narrowband interference filters (IF0, IF1a, IF1b, and IF2) under small angles are shown in Fig. 3.

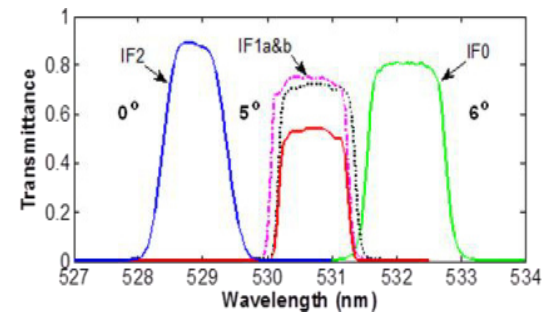

Fig. 3 Transmission curves of the interference filters

\section{RESULTS}

The PRR temperature measurement lidar has been operated for routine observations in the Wuhan institute of physics and mathematics $\left(30.5^{\circ} \mathrm{N}\right.$, $114.4^{\circ} \mathrm{E}$ ) since March 2014. Up to the end of 2014, a total of $\sim 400$ hours of temperature data were collected from more than 50 lidar observational nights.

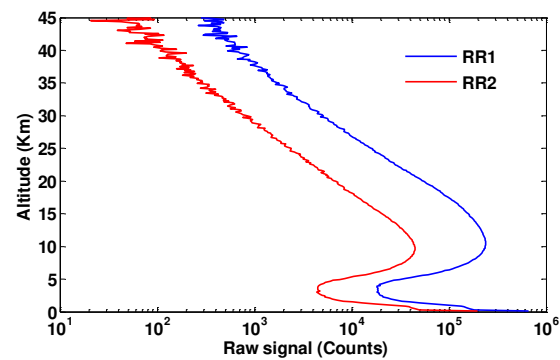

Fig. 4 Raw received signals of the rotational Raman lidar on June 4, 2014.

Fig. 4 exhibits the 1h-integrated signal profiles (background-corrected) received separately from the RR1 and RR2 channels with a range resolution of $150 \mathrm{~m}$. The local time (LT) is 20:00-21:00, June 4, 2014. With the bi-axial configuration of the laser emitter and telescope receiver, the stronger signals at lower altitudes are effectively restrained, which ensures the detection of the weaker signals at higher altitudes.
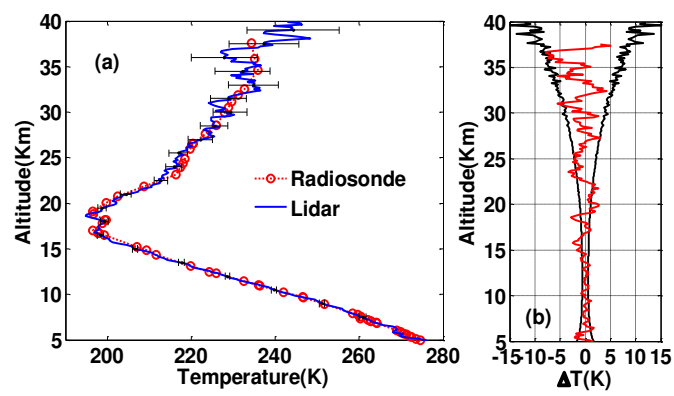

Fig.5 (a) Temperature profiles $(5-40 \mathrm{~km})$ measured by the rotational Raman lidar and local radiosonde. (b) Statistical error and the temperature deviation between lidar and radiosonde on June 4, 2014.

Based on the received signals shown in Fig.4, the temperature profile measured by PRR lidar from $5-40 \mathrm{~km}$ is plotted in Fig. 5 (a) together with the temperature profile measured by the local radiosonde. The launch time for the local radiosonde is 20:00 LT, June 4, 2014, which is close to the lidar measurements in space and time. Fig. 5 (b) shows the statistical temperature error of the lidar measurements as well as the temperature deviation between lidar and radiosonde. The maximum deviation is not more than $3.0 \mathrm{~K}$ for altitudes below $30 \mathrm{~km}$. With the $1-\mathrm{h}$ integration and $150-\mathrm{m}$ spatial resolution, the smallest statistical temperature error is $0.4 \mathrm{~K}$ at $\sim 10 \mathrm{~km}$ where the signal-to-noise level is highest. Below $10 \mathrm{~km}$ there is partial geometric overlap between the laser divergence and the telescope field-of-view, thus the statistical error becomes larger at lower altitudes where the geometric factor (GF) decreases. While the statistical error increases up to $4 \mathrm{~K}$ at $30 \mathrm{~km}$, and above that altitude the error becomes larger as well when the signal-to-noise level lowers. With nearly the same $\mathrm{GF}$, temperature retrieved by two RR channels is obtainable over altitudes of 5-40 km.

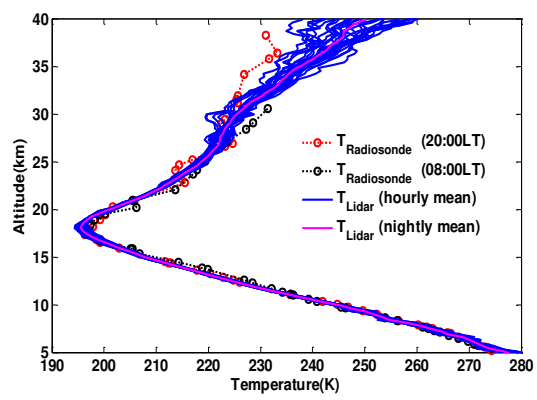

Fig.6 The whole night temperature profiles measured by the rotational Raman lidar and the two local-time radiosondes on August 4-5, 2014.

To achieve smaller temperature error at high altitudes, the temporal or spatial resolution must 
be reduced. Thus a trade-off exists between the measurement precision and the temporal and spatial resolution. Fig. 6 shows the whole night temperature profiles observed on August 4-5, 2014 with 300-m resolution, 1-h integration and 15-min time shift. As the lidar system operates stably, the temperature is obtained with the initial calibration on July 22, 2014. By comparing the lidar-derived temperature with the radiosondes', the good agreement demonstrates that PRR lidar can work continuously and steadily with long term dependability. Besides the given hourly mean temperature profiles (blue lines), the nightly mean temperature shown with the magenta line is also presented. Estimated by subtracting the nightly mean temperature from the individual hour to hour profiles, temperature fluctuations will be observed to study the dynamic processes of the atmosphere.

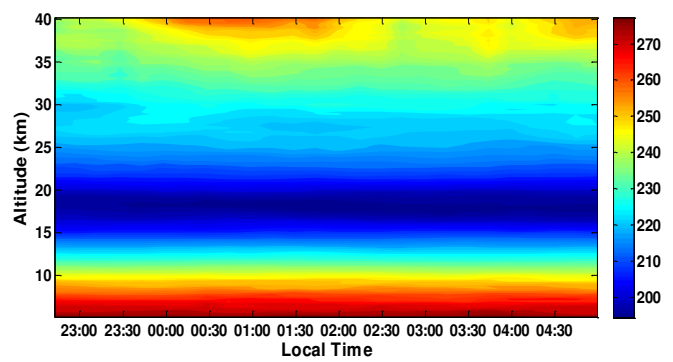

Fig.7 Time-height contour of the temperature profiles observed on August 4-5, 2014

As observed from the time height contour of the temperature profiles during the night shown in Fig. 7, the thermal structure in the lower atmosphere from $5-40 \mathrm{~km}$ is evident with the coldest temperature, $\sim 195 \mathrm{~K}$, at about $18 \mathrm{~km}$.

\section{CONCLUSIONS}

This paper described the system design and observational results of a PRR lidar for the temperature measurements from $5-40 \mathrm{~km}$ over Wuhan. With the high-spectral resolution polychromator for light splitting and filtering, RR scattering returns were detected by weak signal detection technology. Observational results were presented to investigate the overall lidar performance. The temperature profiles simultaneously measured by PRR lidar and the local meteorological radiosonde were compared. The deviation below $30 \mathrm{~km}$ was less than $3.0 \mathrm{~K}$, which validated the reliability of the PRR lidar system. The entire night's temperature profiles demonstrate the long-term capability of the PRR lidar system. With high precision, high temporal and spatial resolution temperature measurements, a more detailed analysis of the fluctuations will allow study of atmospheric dynamical processes. Temperature measurements using PRR lidar combined with Rayleigh lidar will allow an even greater lidar capability.

\section{ACKNOWLEDGEMENT}

We acknowledge the Wuhan Weather Station for providing the radiosonde data. This work was supported by the National Natural Science Foundation of China, under the Grant nos. 41127901, 41101334, 11403085.

\section{REFERENCES}

[1] Alpers, M., R. Eixmann, C. Fricke-Begemann, et al., 2004: Temperature lidar measurements from 1 to $105 \mathrm{~km}$ altitude using resonance, Rayleigh, and Rotational Raman scattering, Atmos. Chem. Phys., 4: 793-800.

[2] Hauchecorne, A., M. L. Chanin, 1980: Density and temperature profiles obtained by lidar between 35 and 70 km, Geophys. Res. Lett., 7: 565-568.

[3] Keckhut, P., M. L.Chanin, A. Hauchecorne, 1990: Stratosphere temperature measurement using Raman Lidar, Appl. Opt., 29(34): 51825186.

[4] Behrendt, A., T. Nakamura, T.Tsuda, 2004: Combined temperature lidar for measurements in the troposphere, stratosphere, and mesosphere, Appl. Opt., 43(14): 2930-2939.

[5] Achtert, P., M. Khaplanov, F.Khosrawi et al., 2013: Pure rotational-Raman channels of the Esrange lidar for temperature and particle extinction measurements in the troposphere and lower stratosphere. Atmos. Meas. Tech., 6: 91-98.

[6] Behrendt, A., 2005: Temperature Measurements with Lidar, Lidar: Range-Resolved Optical Remote Sensing of the Atmosphere. C. E. Weitkamp,ed., Springer, 102: 273-305.

[7] Cooney, J., 1972: Measurement of atmospheric temperature profiles by Raman backscatter, J. Appl. Meteorol., 11: 108-112.

[8] Li, Y., Song, S., Li, F. et al., 2015: High Precision Measurements of lower atmospher ic temperature based on Pure Rotational Raman Li dar, submitted to Chinese J. Geophys. 